\title{
THE NASA AMES PAH IR SPECTROSCOPIC DATABASE AND THE FAR-IR
}

\author{
C. Boersma ${ }^{1}$, L.J. Allamandola ${ }^{1}$, C.W. Bauschlicher, Jr. ${ }^{2}$, A. Ricca ${ }^{2}$, \\ J. Cami ${ }^{3}$, E. Peeters ${ }^{3}$, F. Sánchez de Armas ${ }^{4}$, G. Puerta Saborido ${ }^{4}$, \\ A.L. Mattioda ${ }^{1}$ and D.M. Hudgins ${ }^{5}$
}

\begin{abstract}
Polycyclic Aromatic Hydrocarbons (PAHs) are widespread across the Universe and influence many stages of the Galactic lifecycle. The presence of PAHs has been well established and the rich mid-IR PAH spectrum is now commonly used as a probe into (inter)stellar environments. The NASA Ames PAH IR Spectroscopic Database has been key to test and refine the "PAH hypothesis". This database is a large coherent set ( $>600$ spectra) of laboratory measured and DFT computed infrared spectra of PAHs from $\mathrm{C}_{10} \mathrm{H}_{8}$ to $\mathrm{C}_{130} \mathrm{H}_{28}$ and has been made available on the web at (http://www.astrochem.org/pahdb). With a new spectral window opening up; the far-IR, the study of PAH far-IR spectra and the quest for identifying a unique member of the interstellar PAH family has begun. To guide this research, the far-IR $(>20 \mu \mathrm{m})$ spectra of different sets of PAHs are investigated using the NASA Ames PAH IR Spectroscopic Database. These sets explore the influence of size, shape, charge and composition on the far-IR PAH spectrum. The far-IR is also the domain of the so-called "drumhead" modes and other molecular vibrations involving low order bending vibrations of the carbon skeleton as a whole. As with drums, these are molecule and shape specific and promise to be a key diagnostic for specific PAHs. Here, the sensitivity of these "drumhead" modes to size and shape is assessed by comparing the frequencies of the lowest drumhead modes of a family of circular shaped (the coronene "family") and rhombus shaped (the pyrene "family") PAH molecules. From this study, some consequences for an observing strategy are drawn.
\end{abstract}

\footnotetext{
${ }^{1}$ NASA Ames Research Center, MS 245-6, Moffett Field, CA 94035, USA e-mail: Christiaan.Boersma@nasa.gov

2 NASA Ames Research Center, MS 230-3, Moffett Field, CA 94035, USA

3 Department of Physics and Astronomy, PAB 213, The University of Western Ontario, London, ON N6A 3K7, Canada

${ }^{4}$ SETI Institute, 515 N. Whisman Road, Mountain View, CA 94043, USA

5 NASA Headquarters, MS 3Y28, 300 E St. SW, Washington, DC 20546, USA
} 

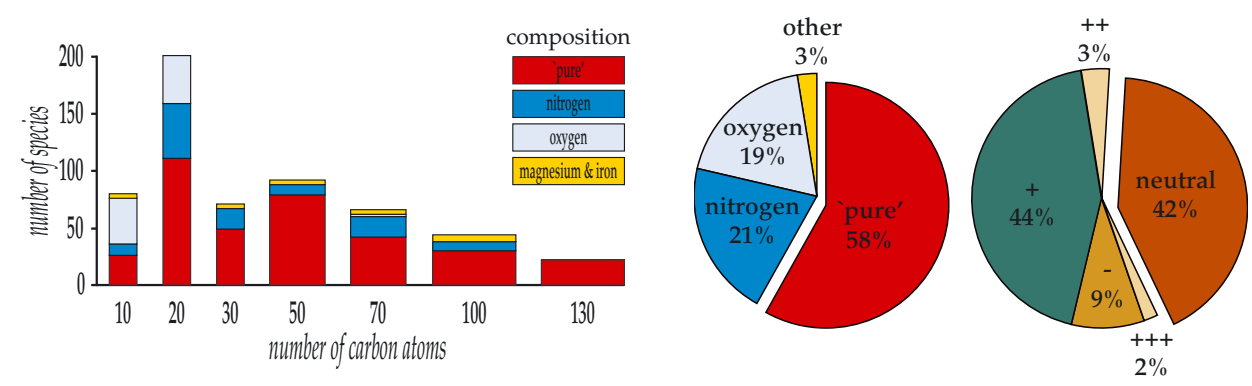

Fig. 1. Left: size distribution, in terms of the number of carbon atoms, of the PAHs in the computational database by composition; "pure" PAHs contain only carbon and hydrogen, nitrogen/oxygen/magnesium \& iron refer to PAHs containing these elements as well. Middle: pie chart showing the breakdown by composition. Right: pie chart showing the charge distribution (Bauschlicher et al. 2010).

\section{Introduction}

The astronomical emission features formerly known as the unidentified infrared bands are now commonly ascribed to polycyclic aromatic hydrocarbons (PAHs). The spectra from laboratory experiments and computational modeling done at the NASA Ames Research Center to test and refine the "PAH hypothesis" have been assembled in a spectroscopic database. Here a few highlights are given from a study of PAH far-IR spectra that utilizes this database (Boersma et al. submitted). This study, that complements that by Mulas et al. (2006) utilizing the Cagliari database of theoretical PAH spectra (Malloci et al. 2007), should prove useful to compare with data obtained by e.g., ESA's Herschel satellite, the Atacama Large Millimeter Array (ALMA), and NASA's Stratospheric Observatory For Infrared Astronomy (SOFIA).

\section{The NASA ames PAH IR spectroscopic database}

The NASA Ames PAH IR Spectroscopic Database now contains over 600 PAH spectra, spanning 2-2000 $\mu \mathrm{m}$, and is available at www.astrochem.org/pahdb. Tools that allow the user to: 1) interrogate the database; 2) plot, compare and co-add spectra, and 3) adjust parameters such as bandwidth etc. have also been made available online. Additionally, the database can be downloaded along with a suite of IDL tools (the AmesPAHdbIDLSuite).

Here the computational data, currently at version 1.11, are utilized and are described in detail by Bauschlicher et al. (2010). The experimental data in the database will be described by Mattioda et al. (in preparation). Figure 1 shows the distribution of the computational database, broken down by size and composition. Figure 1 also summarizes the charge and PAH type distributions in two pie charts.

Due to improvements in computational methods and in computing power, it is now possible to compute the spectra of PAHs containing more than one hundred 
carbon atoms in reasonable amounts of time. When these new sets of spectra become available, they will be added to the database and the version number will be updated accordingly. One promising application is to use the database to directly fit astronomical spectra (see Cami, elsewhere in this volume).

\section{PAH far-IR spectroscopy - some highlights}

Laboratory experiments and DFT computations show that PAH molecules have bands that span the far-IR from 20 to $1000 \mu \mathrm{m}$ and, unlike the mid-IR, where fundamental vibrational frequencies are determined largely by vibrations involving the chemical subgroups and specific bonds which make up the molecule, these longer wavelength transitions originate from vibrations of the entire molecule. Thus, while the mid-IR spectra of PAHs resemble one another since all PAHs are part of the same chemical class, beyond about $15 \mu \mathrm{m}$ band positions can depend on overall size and structure.

\subsection{PAH classes}

The influence of PAH shape, charge, composition and size is studied in Figure 2.

Shape: Comparing the effect of structural modifications on neutral circumcircumcoronene $\left(\mathrm{C}_{96} \mathrm{H}_{24}\right)$ show that with increasing irregularity, far-IR PAH spectra tend to get richer in features.

Charge: The far-IR spectra of $\mathrm{C}_{78} \mathrm{H}_{22}$ in the $-1,0,+,+2$ and +3 charge states shows that varying charge does not have a strong influence on band positions. However, relative band intensities show some differences.

PAHNs: The far-IR spectra of $\mathrm{C}_{96} \mathrm{H}_{24}^{+}$with 8 single nitrogen containing isomers shows that band positions and absolute intensities are hardly affected by the position of the nitrogen atom in the hexagonal network.

Size: The far-IR spectra of ten compact neutral PAHs of increasing size show that the spectra tend to get richer in features and extend further into the far-IR as the molecules get larger.

\subsection{PAH "drumhead" frequencies}

Figure 3 presents the lowest vibrational mode of four members of both the coronene and pyrene "families". The plot demonstrates the shift to lower frequencies of the so-called "drumhead" mode as molecular size increases. The vibrational frequencies are well fitted by:

$$
\nu=600\left(\frac{10^{-15}\left[\mathrm{~cm}^{2}\right]}{A}\right)\left[\mathrm{cm}^{-1}\right],
$$

claiming an inverse dependence of frequency on PAH area $(A)$ for the compact PAHs and only a weak dependence on molecular geometry; circular versus rhombus shaped. 

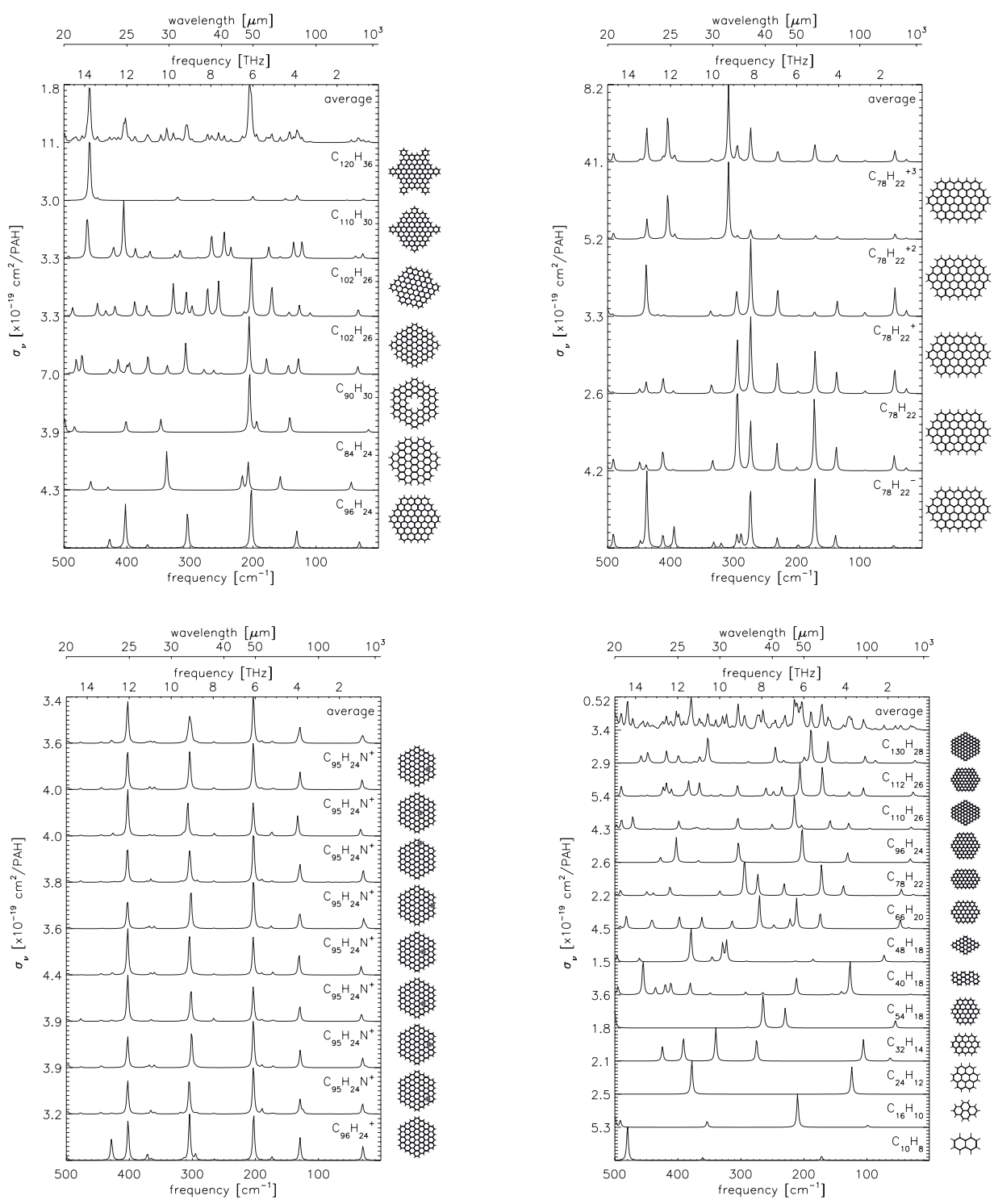

Fig. 2. Series of far-IR PAH spectra $(20-1000 \mu \mathrm{m})$ showing, from top-left-to-bottomright, the effect of shape, charge, nitrogen substitution, and size. Bands have been given Lorentzian profiles with a FWHM of $6 \mathrm{~cm}^{-1}$. See Boersma et al. (submitted) for a detailed discussion.

Considering PAH molecules as a solid plate, this is perhaps not that surprising. The classic solution for the frequency of vibration of "free" plates is given by 

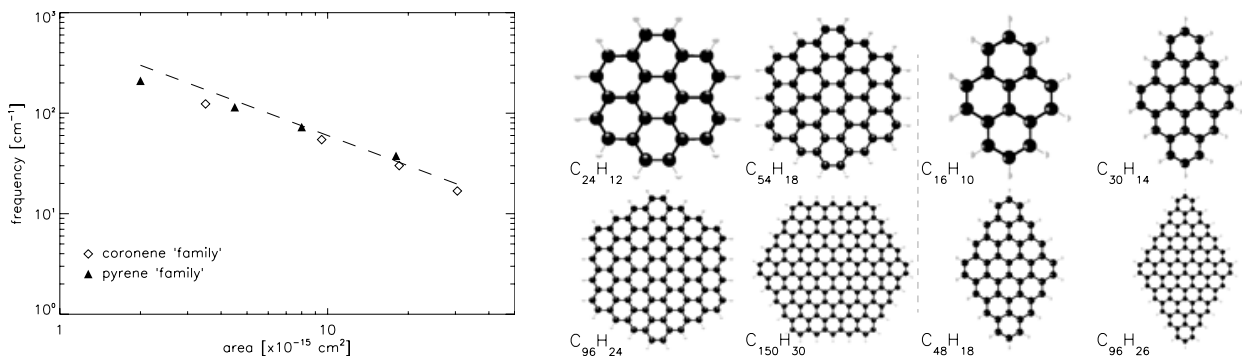

Fig. 3. Left: comparison of the predicted $(0,1)$ frequencies of a solid graphene plate, Equation (3.2), with those found in the database for the coronene (squares) and pyrene (triangles) "families". Right: members of the coronene and pyrene "families". From Boersma et al. (submitted).

(Meirovitch 1997):

$$
\nu=\frac{\pi}{2 c} \frac{1}{A} \sqrt{\frac{D}{\rho h}} \cdot\left(n^{2}+m^{2}\right),
$$

with $c$ the speed of light, $A$ the surface area, $\rho$ the density, $h$ the plate thickness and $D$ the rigidity. The modes are characterized by $m$ and $n$; the number of nodes along both plate-axes. Because of fundamental and commercial interest, there is a rich literature on the mechanical properties of graphene and carbon nano-tubes. The bending rigidity of a graphene sheet has been calculated to be $0.8-1.5 \mathrm{eV}$, depending on the method used (Salvetat et al. 2006). Adopting $D=1.5 \mathrm{eV}$ and $7.5 \times 10^{-8} \mathrm{~g} \cdot \mathrm{cm}^{-2}$ for the surface density $(\rho h)$, the data are well reproduced.

\subsection{PAH temperature}

The far-IR absorption band strengths are generally an order of magnitude smaller than the mid-IR absorption band strengths. However in the common astronomical case of emission, relative band strengths can change significantly depending on the PAH's internal vibrational energy (temperature).

In the framework of the thermal model, conservation of energy leads to the following expression:

$$
4 \pi \sum_{i} \sigma_{i} \int_{T_{i}}^{T_{\max }} B\left(\nu_{i}, T\right)\left[\frac{\mathrm{d} T}{\mathrm{~d} t}\right]^{-1} \mathrm{~d} T=E_{\text {internal }}
$$

where $\sigma_{i}$ is the absorption cross-section in mode $i ; B\left(\nu_{i}, T\right)$, is Planck's function at frequency $\nu_{i}$ in mode $i$, at temperature $T$; $\mathrm{d} T / \mathrm{d} t$ is the cooling-rate and $E_{\text {internal }}$ is the PAH's internal vibrational energy after excitation by a FUV photon. The sum is taken over all modes and the integral runs from the initial PAH temperature up to the PAH's maximum attained temperature $\left(T_{\mathrm{i}}-T_{\max }\right)$ after absorption of the exciting photon. 


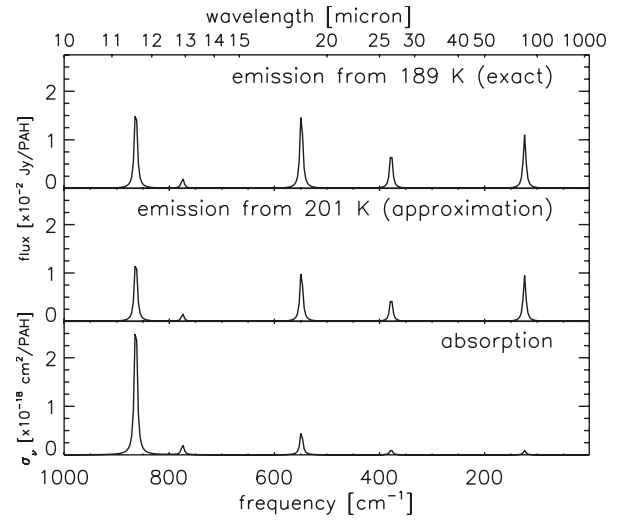

Fig. 4. Comparison of the synthesized absorption spectrum $(10-1000 \mu \mathrm{m})$ of coronene (bottom) with the emission spectrum produced with an internal vibrational energy of $0.1 \mathrm{eV}$, both when approximating the calculation of the heat capacity (middle) and exactly calculating the heat capacity (top; see also Boersma et al. submitted). Bands have been given Lorentzian profiles with a FWHM of $6 \mathrm{~cm}^{-1}$.

The maximum attained temperature is directly related to the energy of the absorbed photon through the PAH heat capacity, which also effects its coolingrate. The heat capacity can be calculated exactly by treating the PAH molecule as a molecular system of isolated harmonic oscillators or approximated (see e.g., Dwek et al. 1997).

Applying the thermal model to neutral coronene with an internal vibrational energy of $0.1 \mathrm{eV}$ results in a maximum temperature of 189 Kelvin when calculating the full heat capacity exactly and 201 Kelvin when using the approximation. Comparing both methods in Figure 4 shows the approximation is reasonably.

In absorption the coronene "drumhead" band intensity at $124 \mathrm{~cm}^{-1}$ is $2.9 \%$ of that of the strongest band at $863 \mathrm{~cm}^{-1}$, whereas at $201 \mathrm{~K}$ it has $60 \%$ of the intensity of the band at $863 \mathrm{~cm}^{-1}$. However, it should be kept in mind that the intrinsic strength of these bands is still weak.

\subsection{Observing PAHs in the far-IR}

Far-IR spectra of astronomical objects showing the mid-IR PAH emission features will provide a very different perspective on the PAH population than do the midIR bands, e.g., local conditions, chemical history of carbonaceous material, etc. Because the far-IR probes much colder material than the mid-IR, these data will explore the large end of the astronomical PAH-size-distribution and thus expand the scope of astronomical and astrochemical regimes in which PAHs can serve as probes.

The far-IR bands promise to put a firm limit on the size of the emitting PAHs. However, the molecular structure of the PAH does not play a major part in determining the far-IR spectrum and, therefore, identifying specific molecules in the interstellar PAH family likely requires other means. Mulas et al. (2006) point to the rotational PQR band structure of the lowest PAH mode as such a means. Joblin et al., elsewhere in this volume, address an observational strategy based on this band structure. 
Calculations have shown that the lowest vibrational transitions of interstellar PAHs probably contain a few tenths of a percent of the absorbed FUV energy (Mulas et al. 2006). While this is only a tiny fraction, the decrease in background dust continuum emission expected for warm PDRs could enhance the spectroscopic contrast. One first-look observing strategy could be to study the emission from transition zones in harsh environments. Telescope sensitivity will then not be an issue and UV photolysis may have isomerised the PAHs to their most stable form, leaving only a very limited number of different species. Given their high stability and likely contribution to the mid-IR (Bauschlicher et al. 2008; Mattioda et al. 2009), the presented coronene and pyrene "families" are particularly interesting in this regard.

\section{References}

Bauschlicher, C.W., Boersma, C., Ricca, A., et al., 2010, ApJS, 189, 341

Bauschlicher, C.W., Peeters, E., \& Allamandola, L.J., 2008, ApJ, 678, 316

Boersma, C., Bauschlicher, C.W., Ricca, A., et al., ApJ, submitted

Dwek, E., Arendt, R.G., Fixsen, D.J., et al., 1997, ApJ, 475, 565

Malloci, G., Joblin, C., \& Mulas, G., 2007, Chemical Phys., 332, 353

Mattioda, A.L., Hudgins, D.M., Boersma, C., et al., in preparation

Mattioda, A.L., Ricca, A., Tucker, J., Bauschlicher, C.W., \& Allamandola, L.J., 2009, ApJ, 137, 4054

Meirovitch, L., 1997, Principles and techniques of vibrations (Prentice-Hall International)

Mulas, G., Malloci, G., Joblin, C., \& Toublanc, D., 2006, A\&A, 460, 93

Salvetat, J.P., Désarmot, G., Gauthier, C., \& Poulin, P., 2006, Lecture Notes in Physics, Vol. 677, Mechanical Properties of Individual Nanotubes and Composites (Springer Berlin/Heidelberg), 439 